\title{
Evaluation of the yield of post-clipping angiography and nationwide current practice
}

\author{
N. Scheer ${ }^{1} \cdot$ R. Ghaznawi ${ }^{1}$ - M. A. A. van Walderveen ${ }^{2} \cdot$ R. W. Koot ${ }^{1} \cdot$ P. W. A. Willems ${ }^{3}$
}

Received: 27 November 2018 / Accepted: 31 January 2019 /Published online: 19 February 2019

(C) The Author(s) 2019

\begin{abstract}
Background Surgical treatment of intracranial saccular aneurysms aims to prevent (re)hemorrhage by complete occlusion of the aneurysmal lumen. It is unclear whether routine postoperative imaging, to assess aneurysmal occlusion, is necessary since intraoperative assessment by the neurosurgeon may be sufficient. We assessed routine clinical protocols for post-clipping imaging in the Netherlands and determined whether intraoperative assessment of aneurysm clippings sufficiently predicts aneurysm residuals.

Methods A survey was conducted to assess postoperative imaging protocols in centers performing clipping of intracranial aneurysms in the Netherlands $(n=9)$. Furthermore, a retrospective single-center cohort study was performed to determine the predictive value of intraoperative assessment of aneurysm occlusion in relation to postoperative digital subtraction angiography (DSA) findings, between 2009 and 2017.

Results No center performed intraoperative DSA in a hybrid OR, routinely. Respectively, four (44.4\%), seven (77.8\%), and three (33.3\%) centers did not routinely perform early postoperative imaging, late follow-up imaging, or any routine imaging at all. Regarding our retrospective study, 106 patients with 132 clipped aneurysms were included. There were 23 residuals $\geq 1 \mathrm{~mm}$ $(17.4 \%)$, of which $10(43.5 \%)$ were unexpected. For the presence of these residuals, intraoperative assessment showed a sensitivity of $56.5 \%$, a specificity of $86.2 \%$, a positive predictive value of $46.4 \%$, and a negative predictive value of $90.4 \%$.

Conclusions There is lack of consensus regarding the post-clipping imaging strategy in the Netherlands. Since intraoperative assessment is shown to be insufficient to predict postoperative aneurysm residuals, we advocate routine postoperative imaging after aneurysm clipping unless this is not warranted on the basis of patient age, clinical condition, and/or comorbidity.
\end{abstract}

Keywords Saccular aneurysm $\cdot$ Ruptured $\cdot$ Unruptured $\cdot$ Surgery $\cdot$ Residual $\cdot$ Retreatment

\section{Introduction}

Subarachnoid hemorrhage (SAH) is a severe subtype of stroke. Not only does it carry significant morbimortality [21, 28], it also affects patients at a mean age of 57 years [21]

This article is part of the Topical Collection on Vascular Neurosurgery Aneurysm

P. W. A. Willems

p.w.a.willems-4@umcutrecht.nl

1 Department of Neurosurgery, Leiden University Medical Center, Leiden, the Netherlands

2 Department of Radiology, Leiden University Medical Center, Leiden, the Netherlands

3 Department of Neurosurgery, University Medical Center Utrecht, Internal Postage: G03.124, PO-box 85500, 3584

CX Utrecht, The Netherlands leading to loss of many years of productive life. Intracranial ruptured aneurysms are the underlying cause in $85 \%$ of patients [21]. Treatment of intracranial saccular aneurysms, with or without prior SAH, primarily aims to prevent (re)bleeding by complete occlusion of the aneurysmal lumen. The current treatment of intracranial aneurysms consists of microsurgical clipping or endovascular treatment (coiling). Although endovascular treatment is performed in the majority of patients, microsurgical clipping still has some advantages and is the preferred treatment in a selective group of patients [14]. Successful clipping is defined as the complete and permanent exclusion of the entire aneurysm from the arterial circulation. The necessity for post-clipping imaging depends on the level of certainty, intraoperatively, whether complete occlusion has been achieved.

Post-clipping imaging, if necessary, is most accurate with digital subtraction angiography (DSA) [26]. It is considered the gold standard to identify residual filling of the aneurysmal 
lumen, which may necessitate additional follow-up and/or treatment [20]. However, performing DSA routinely adds risk [29] and burden to a majority of patients whose aneurysms prove to be adequately obliterated. A less invasive alternative is computerized tomographic angiography (CTA), but this imaging technique has been shown to be less sensitive [25]. With the number of reoperations being small [5], no consensus currently exists regarding the implementation of routine postoperative imaging, either DSA or CTA.

We hypothesized that the surgeon's intraoperative assessment is sufficient to determine aneurysm occlusion. If so, this would render routine postoperative DSA superfluous (or intraoperative DSA in case of a hybrid operating room). To address this issue, we surveyed current routine clinical protocols for post-clipping imaging in the Netherlands. Furthermore, we performed a retrospective cohort study to determine to which extent intraoperative assessment of aneurysmal occlusion was in agreement with postoperative DSA results.

\section{Methods}

\section{National survey regarding the use of imaging}

To assess routine clinical protocols for post-clipping imaging in the Netherlands, a questionnaire was sent to a vascular neurosurgeon from each of the nine centers in the Netherlands performing microsurgical clipping of aneurysms, in 2017. In this survey, the neurosurgeons were asked what the general strategy at their center was (1) regarding early postoperative imaging ( $<90$ days post clipping) and (2) regarding late follow-up imaging ( $>90$ days post clipping). For both questions, they could choose from the following answers: (a) routine DSA, (b) routine CTA, (c) DSA if indicated, (d) CTA if indicated, or (e) no imaging. If the neurosurgeon chose option $\mathrm{c}$ or $\mathrm{d}$ (imaging if indicated) regarding early postoperative imaging, they were asked to specify the indication(s) for performing imaging.

In December of 2018, the same vascular neurosurgeons were asked whether their center had a hybrid operating room and, if so, whether it was routinely used to perform intraoperative DSA during or after aneurysm clipping?

\section{Retrospective study evaluating intraoperative assessment}

\section{Research design and inclusion criteria}

We performed a retrospective single-center cohort study, including all patients with either a ruptured or unruptured aneurysm, who were treated microsurgically at our center, between January 2009 and August 2017, and who underwent a postoperative DSA within 90 days after surgery. Year 2009 was chosen as starting point as post-clipping DSA was not routine in our center until that time. Since then, its use leveled off at $68 \%$ of the cases, on average.

\section{Data collection}

Clinical information was obtained from electronic and paper medical records. The collected information included patient demographics (age, gender), risk factors for aneurysm formation (hypertension, tobacco abuse, alcohol abuse, drug abuse, positive family history of intracranial aneurysms, history of $\mathrm{SAH}$ ), aneurysm location, aneurysm rupture, timing of aneurysm surgery (emergency or elective setting), rebleeds, timing of postoperative DSA, and clinical consequence of postoperative DSA. In patients with multiple aneurysms, each aneurysm being clipped was noted as a separate event. In cases of $\mathrm{SAH}$, patient admission status was reconstructed from the records, using the Hunt and Hess (HH) scale and the World Federation of Neurosurgical Societies (WFNS) scale.

Postoperative DSA was performed with standard biplane fluoroscopy equipment (Infinix, Toshiba Medical Systems, Japan) and consisted of at least anteroposterior, lateral, and rotational images of the territory concerned, including $3 \mathrm{D}$ reconstructions. The DSA report of every clipped aneurysm was used to identify aneurysm residuals. The size of all residuals was subsequently measured by a neurosurgeon co-specialized in neuro-interventional radiology (PWAW).

\section{Definitions of outcome measures}

Operative reports were reviewed to determine whether the neurosurgeon expected a postoperative residual or not. Each report was categorized as one of the following: "not expecting a residual" (ExpN), "expecting a residual" (ExpY), or "inconclusive" (ExpI). Surgical reports mentioning complete aneurysm occlusion as well as reports with no remark about completeness of aneurysm occlusion but without any remarks regarding difficulties during surgery, were categorized as ExpN. Surgical reports specifically mentioning incomplete occlusion were categorized as ExpY. Those mentioning difficulties during surgery but without a statement on the final result were categorized as ExpI. To avoid underestimation of the number of unexpected residuals, we dichotomized between ExpN on the one hand and either ExpY or ExpI on the other in the final analysis.

Residual aneurysms at DSA were categorized as follows: no residual $(R=0)$, residual $<1 \mathrm{~mm}(R<1)$ and residual $\geq$ $1 \mathrm{~mm}(R \geq 1)$. In the final analysis, these categories were dichotomized as $R \geq 1$ on the one hand and either $R<1$ or $R=0$ on the other, because residuals $\geq 1 \mathrm{~mm}$ have a higher risk of regrowth [6] and are, therefore, clinically relevant. 


\section{Statistical analysis}

All analyses were performed using Statistical Package for the Social Sciences (SPSS) version 20. Normally distributed variables were expressed as mean with standard deviation (SD) and/or range, when appropriate. Skewed distributed variables were expressed as median with interquartile range (IQR). Statistical significance was defined as $P<0.05$.

Sensitivity, specificity, positive, and negative predictive values with their $95 \%$ confidence intervals (CI) were determined for the intraoperative assessment. DSA results acted as gold standard.

Imaging is most likely to be omitted in cases when a residual is not expected (ExpN). Therefore, we decided to perform an additional analysis in this subgroup to determine whether other variables could predict the need for DSA imaging. These variables included aneurysm location, timing of aneurysm surgery, HH scale and WFNS scale. For this analysis, the variable aneurysm location was dichotomized as "aneurysm at anterior communicating or ophthalmic artery region" versus "aneurysm at other location," since deep midline aneurysms and aneurysms at an ophthalmic artery region have an increased risk of imperfect clip placement [23].

\section{Results}

\section{National survey regarding intraoperative and postoperative imaging}

The response rate of the survey was $100 \%$ (9/9).

Regarding a hybrid operating room, only five centers $(55.6 \%)$ had one at the beginning of 2019 and at least two of these had only recently become available. Three other centers were in the process of building one. None of the centers used the hybrid room routinely for aneurysm clipping, and only one center used it routinely for unruptured aneurysms.

The responses regarding postoperative imaging are shown in Fig. 1. One neurosurgeon answered "DSA if indicated" on the question regarding early postoperative imaging. The neurosurgeon explained that performing DSA in his center depended on the intra-operative impression of the neurosurgeon regarding a possible residual. Four centers did not routinely perform early postoperative imaging (44.4\%) and seven did not routinely perform late imaging $(77.8 \%)$. Three centers performed neither routine early nor routine late postoperative imaging (33.3\%).

\section{Retrospective study evaluating intraoperative assessment}

\section{Patient population}

We identified 160 patients with 198 ruptured and unruptured aneurysms, clipped between January 2009 and August 2017. Thirty-seven patients $(23.1 \%)$ with 40 aneurysms $(20.2 \%)$ were excluded, due to lack of a postoperative DSA. Reasons for not performing an angiography were as follows: poor clinical condition (20 patients), relocation to primary institution prior to DSA (5 patients), DSA deemed too risky (1 patient), renal failure (1 patient), DSA refused by the patient (1 patient), or an unknown reason ( 9 patients). This resulted in 123 patients, with 158 aneurysms. Of these patients, 106 cases (86.2\%) with 132 aneurysms $(83.5 \%)$ underwent angiography within 90 days post clipping. These patients were included in the study. The baseline characteristics of the patients are shown in Table 1. The characteristics of the 132 clipped aneurysms are presented in Table 2. The median time to DSA was 5.0 days after surgery (IQR 2.3-9.0 days). The majority of patients (68.9\% of all aneurysms) had a postoperative DSA within 1 week after clipping.

\section{Residuals}

Evaluation of DSA imaging of the 132 clipped aneurysms yielded 23 aneurysms labeled $R \geq 1$ (17.4\%), 11 aneurysms with $R<1(8.3 \%)$, and 98 aneurysms with $R=0$ (74.2\%). Characteristics of the $R \geq 1$ aneurysms are shown in Table 3 .
Fig. 1 Pie charts of the responses on the national survey regarding postoperative imaging $(n=9)$. Left: early postoperative imaging (<90 days post clipping). Right: late follow-up imaging ( $>90$ days post clipping). CTA computerized tomographic angiography; DSA (or 'angiography') digital subtraction angiography
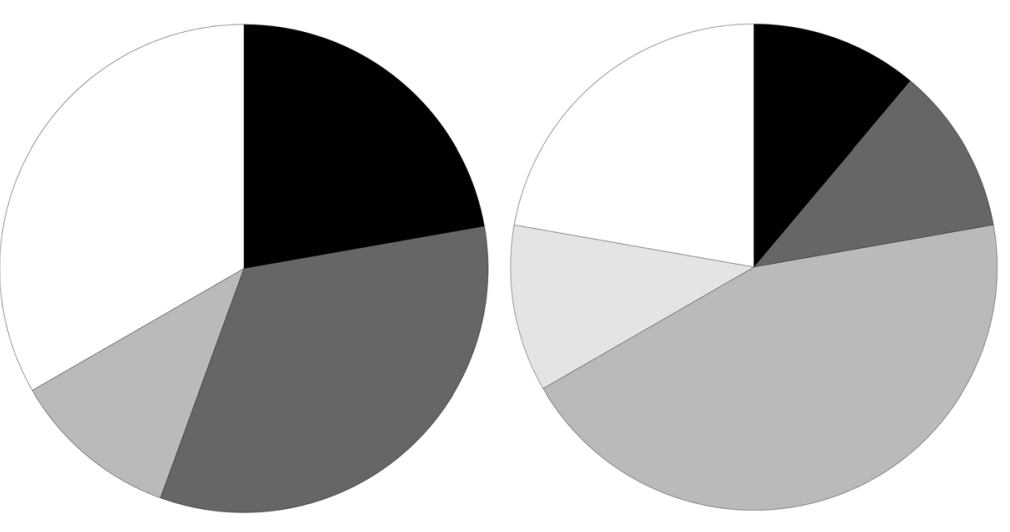

Responses of the centers Routine angiography Routine CTA DSA if indicated CTA if indicated No imaging 
Table 1 Patient characteristics $(n=106$ patients)

\begin{tabular}{|c|c|}
\hline Characteristic & Value $(\%)$ \\
\hline \multicolumn{2}{|l|}{ Age (years) } \\
\hline Mean \pm SD & $55.3 \pm 13.2$ \\
\hline Range & $12-79$ \\
\hline Gender male & $26(24.5)$ \\
\hline Presenting with SAH & $69(65.1)$ \\
\hline \multirow[t]{5}{*}{ WFNS grade at arrival in hospital } & $1: 39(56.5)$ \\
\hline & 2: $8(11.6)$ \\
\hline & $3: 3(4.3)$ \\
\hline & 4: 8 (11.6) \\
\hline & 5: $11(15.9)$ \\
\hline \multirow[t]{5}{*}{$\mathrm{HH}$ grade at arrival in hospital } & 1: $15(21.7)$ \\
\hline & $2: 27(39.1)$ \\
\hline & 3: $11(15.9)$ \\
\hline & $4: 10(14.5)$ \\
\hline & 5: $6(8.7)$ \\
\hline \multicolumn{2}{|l|}{ Number of clipped aneurysms per patient } \\
\hline One aneurysm & $84(79.2)$ \\
\hline Two aneurysms & $18(17.0)$ \\
\hline Three aneurysms & $4(3.8)$ \\
\hline \multicolumn{2}{|l|}{ Risk factors aneurysm formation } \\
\hline Hypertension & $40(37.7)$ \\
\hline Tobacco abuse & $41(38.7)$ \\
\hline Alcohol abuse & $12(11.3)$ \\
\hline Drug abuse & $4(3.8)$ \\
\hline Positive family history of intracranial aneurysms & $6(5.7)$ \\
\hline History of SAH & $3(2.8)$ \\
\hline
\end{tabular}

$S A H$, subarachnoid hemorrhage; WFNS, World Federation of Neurosurgical Societies; $H H$, Hunt and Hess

\section{Evaluation of intraoperative assessment and postoperative angiography}

Of the 23 residuals $\geq 1 \mathrm{~mm}$ in size, 10 were expected $(43.5 \%)$ based on the intraoperative assessment, 10 were unexpected (43.5\%), and in 3 the assessment was inconclusive (13.0\%). The results of the intraoperative assessments and postoperative angiographies are shown in Table 4.

The sensitivity of the intraoperative assessment for the presence of a residual $\geq 1 \mathrm{~mm}$ was $56.5 \%(95 \% \mathrm{CI}=$ $0.35-0.76)$, its specificity $86.2 \%(95 \% \mathrm{CI}=0.78-0.92)$, its positive predictive value $46.4 \%(95 \% \mathrm{CI}=0.28-$ $0.66)$, and its negative predictive value $90.4 \%(95 \%$ $\mathrm{CI}=0.83-0.95)$.

\section{Clinical consequences of the residuals}

The clinical consequences of finding a residual on postoperative DSA were as follows: follow-up DSA in 18 aneurysms
Table 2 Aneurysm characteristics $(n=132$ aneurysms $)$

\begin{tabular}{ll}
\hline Characteristic & Value $(\%)$ \\
\hline Location & \\
ACA (excluding ACoA) & $9(6.8)$ \\
AChA & $8(6.1)$ \\
ACoA & $20(15.2)$ \\
ICA & $6(4.5)$ \\
MCA & $67(50.8)$ \\
PCoA & $18(13.6)$ \\
PICA & $3(2.3)$ \\
VA & $1(0.8)$ \\
Ruptured & $66(50.0)$ \\
Unruptured & $66(50.0)$ \\
Of which clipped in emergency setting & $23(34.8)$ \\
Rebleed after clipping & 0
\end{tabular}

$A C A$, anterior cerebral artery; $A C h A$, anterior choroidal artery; $A C o A$, anterior communicating artery; $I C A$, internal carotid artery; $M C A$, middle cerebral artery; $P C O A$, posterior communicating artery; $P I C A$, posterior inferior cerebellar artery; $V A$, vertebral artery

(78.3\%), endovascular re-treatment (coiling) in 1 aneurysm (4.3\%), surgical re-treatment in 1 aneurysm (4.3\%), and no consequence in 3 aneurysms (13.0\%).

When these consequences were dichotomized on the basis of whether the residual had been expected, no obvious differences were perceived. Out of 10 residuals that were labeled ExpN, 8 had a follow-up DSA (80\%), and one was retreated endovascularly (10\%), while out of 13 residuals that were labeled either ExpY or ExpI, 10 had a follow-up DSA (77\%), and one was retreated surgically $(8 \%)$.

Table 3 Characteristics of residuals $\geq 1 \mathrm{~mm}(n=23)$

\begin{tabular}{ll}
\hline Characteristic & Value (\%) \\
\hline Location & \\
AChA & $2(8.7)$ \\
ACoA & $7(30.4)$ \\
MCA & $9(39.1)$ \\
PCoA & $5(21.7)$ \\
Size & \\
Median (IQR) & $1.5(1.2-2.5)$ \\
Range & $1.0-14.0$ \\
Ruptured aneurysm & $17(73.9)$ \\
Unruptured aneurysm & $6(26.1)$ \\
Of which clipped in emergency setting & $2(33.3)$ \\
\hline
\end{tabular}

$A C h A$, anterior choroidal artery; $A C O A$, anterior communicating artery; $M C A$, middle cerebral artery; $P C O A$, posterior communicating artery; $I Q R$, interquartile range 
Table 4 Results of the operative reports and postoperative DSA ( $n=$ 132)

\begin{tabular}{lllll}
\hline & & \multicolumn{2}{l}{ Postoperative DSA } & \multirow{2}{*}{ Total } \\
\cline { 3 - 4 } & & $R \geq 1$ & $R=0$ or $R<1$ & \\
\hline \multirow{2}{*}{ Operative report } & ExpY or ExpI & 13 & 15 & 28 \\
& ExpN & 10 & 94 & 104 \\
& Total & 23 & 109 & 132 \\
\hline
\end{tabular}

$D S A$, digital subtraction angiography; ExpI, an operative report categorized as "inconclusive;" $\operatorname{ExpN}$, is an operative report categorized as "not expecting a residual;" ExpY, an operative report categorized as "expecting a residual;" $R=0$, no residual, $R<1$, residual $<1 \mathrm{~mm}, R \geq 1$, residual $\geq$ $1 \mathrm{~mm}$

\section{Subgroup analysis}

In the ExpN subgroup, logistic regression analysis did not reveal significant associations between timing of aneurysm surgery $(P=0.06)$, location $(P=0.41)$, HH scale $(P=0.21)$ or WFNS scale $(P=0.52)$ and $R \geq 1$. In other words, the occurrence of an aneurysm residual of $1 \mathrm{~mm}$ or larger that was not to be expected from the intraoperative assessment was not associated with any of these parameters.

\section{Case presentations}

We present details of the two cases with residuals requiring retreatment.

Case 1. A 54-year-old woman presented with SAH, WFNS grade 1. DSA revealed a $3.7 \mathrm{~mm}$, broad-based, posterior communicating artery (PCoA) aneurysm. The aneurysm was clipped and, based on the operative report, the neurosurgeon did not expect a residual. After the operation, the patient suffered from bradyphrenia due to vasospasm, for which induced hypertension was initiated on the intensive care unit. Postoperative DSA was performed 5 days later, showing residual filling of the aneurysm and severe angiographic vasospasm. The residual aneurysm was $2.3 \mathrm{~mm}$ in size $(R \geq 1)$. Within 2 days, the aneurysm was successfully coiled. DSA images of this patient are shown in Fig. 2.

Case 2. A 67-year-old woman arrived in the hospital with $\mathrm{SAH}$, WFNS grade 1. CTA revealed a lobulated, $14.0 \mathrm{~mm}$, PCoA aneurysm in the context of a fetal origin of the posterior cerebral artery. The aneurysm was clipped. In his operative report, the neurosurgeon reported multiple problems during surgery and finally described "almost complete occlusion" of the aneurysm. Surprisingly, postoperative DSA demonstrated complete, albeit slowed, filling of the entire aneurysm through a small remaining channel at the level of the aneurysm neck. Successful re-clipping followed the next day. Key images are shown in Fig. 3.

\section{Discussion}

We aimed to assess routine clinical protocols regarding imaging after intracranial aneurysm clipping in the Netherlands and to determine the predictive value of the intraoperative assessment for a postoperative residual. In a national survey, clinical protocols varied widely. In a single-center retrospective evaluation, the negative predictive value of intraoperative assessment was shown to be only $90.4 \%$.

\section{Routine clinical protocols for post clipping imaging}

Almost half of the Dutch centers treating intracranial aneurysms (4/9) did not routinely perform early postoperative imaging and a third (3/9) performed neither routine early nor routine late imaging. Apparently, these centers expected
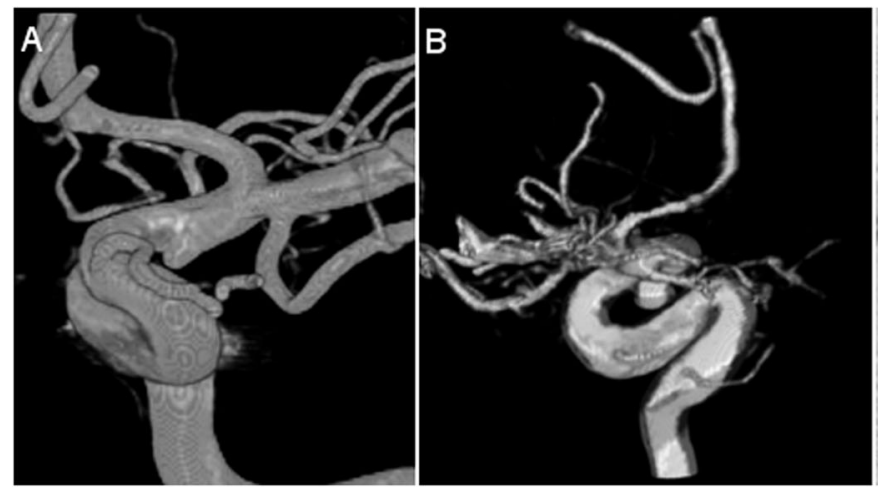

Fig. 2 DSA images of a patient with a postoperative residual requiring coiling. a Preoperative 3D-reconstruction of the rotational angiogram demonstrating a ruptured, $3.7 \mathrm{~mm}$, left PCoA aneurysm. b Postoperative $3 \mathrm{D}$-reconstruction of the rotational angiogram showing residual filling. c DSA (lateral projection) showing the result of coiling. DSA digital subtraction angiography, PCoA posterior communicating artery 

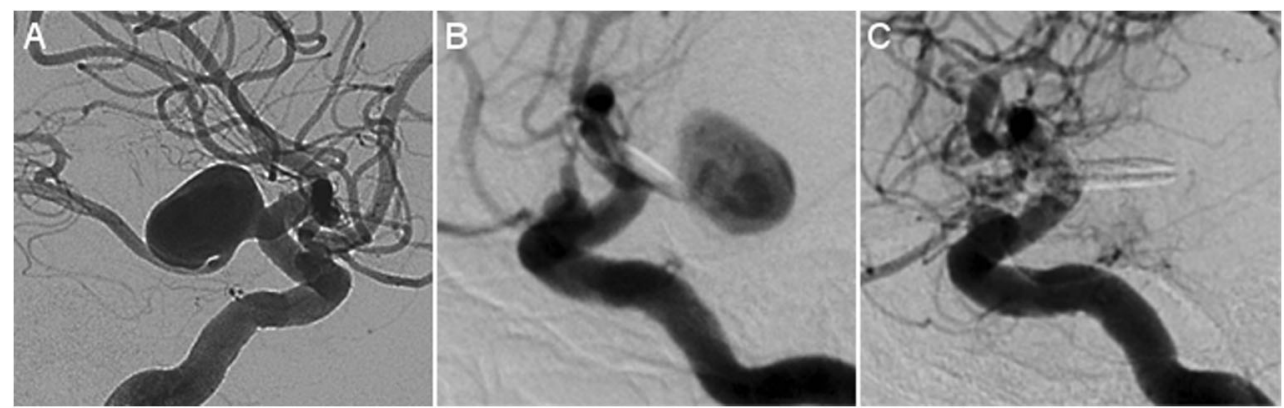

Fig. 3 DSA images of a patient with a postoperative residual requiring reclipping. a DSA (lateral projection) demonstrating a ruptured, $14.0 \mathrm{~mm}$, PCoA aneurysm. b Postoperative DSA (lateral projection) showing

incomplete aneurysmal occlusion to be evident during surgery, in contrast to our current findings. Of the five centers performing routine early postoperative imaging, three used CTA and two used DSA. This represents the debate in literature regarding the optimal type of imaging for clipped aneurysms. Multiple studies suggest that postoperative assessment of clipped aneurysms can be performed with CTA, as an alternative for DSA [7, 8, 12], or suggest to limit the use of DSA to certain indications $[10,17,27]$. However, a recent metaanalysis, with a total of 487 aneurysms, showed that CTA had a pooled sensitivity of only $71 \%$ and specificity of $94 \%$ for identifying residual or recurrent aneurysms in comparison to DSA [25]. For aneurysm residuals $<2 \mathrm{~mm}$, the sensitivity has been shown to be even lower [26]. Although residuals $<1 \mathrm{~mm}$ have been shown to have a lower regrowth risk [6], all residuals $\geq 1 \mathrm{~mm}$ should be considered clinically relevant for risk of regrowth and hemorrhage [6, 13]. Taking this into account, we believe an optimal imaging strategy should include at least one DSA.

When a hybrid OR is available, immediate intraoperative post clipping DSA could substitute for postoperative imaging, but our results clearly indicate that this is not yet routine practice in our country.

\section{Predictive value of intraoperative assessment}

The residual rate after clipping, either expected or unexpected, was $17.4 \%$ in our series. This is slightly higher compared to previous studies (Table 5). With regard to the operative reports, which we regard to be an indicator of the surgeons intraoperative impression, the predictive value for a postoperative residual was very limited. The negative predictive value of the intraoperative assessment for a clinically relevant residual was only $90.4 \%$. This implies that of all the cases where the surgeon would likely find postoperative imaging unnecessary, almost $10 \%$ would still contain a residual. Apparently, it is not straightforward for a surgeon to assess complete aneurysm occlusion. This is in accordance with previous studies $[16,19,20]$. However, our results are relevant since the study from MacDonald et al. [19] dates from before the use of residual filling of the entire aneurysm. c DSA (lateral projection) showing successful re-clipping. DSA digital subtraction angiography, PCoA posterior communicating artery

rotational angiography, Kivisaari et al. [16] did not clarify how the distinction 'unexpected' was made and Meyer et al. [20] found a much lower incidence of unexpected residuals. Moreover, the fact that one of our unexpected residuals required early re-treatment, also suggests that routine postoperative imaging is important.

\section{Study limitations}

With regard to the survey, we asked one neurosurgeon from each center to describe the general strategy at that center. This may lead to a slight misrepresentation as surgeons within one center may also use different imaging strategies. However, this would only further underline the lack of consensus and need for an evidence based guideline.

Table 5 Publications of postoperative residuals on digital subtraction angiography

\begin{tabular}{lcc}
\hline Authors and year & Aneurysms & $\%$ Residuals \\
\hline Acevedo et al. (1997) [1] & 267 & 6.3 \\
Akyüz et al. (2002) [2] & 186 & 7.0 \\
Akyüz et al. (2004) [3] & 166 & 4.2 \\
Bernat et al. (2017) [4] & 37 & $24.3 *$ \\
Brown et al. (2017) [5] & 758 & 7.8 \\
Burkhardt et al. (2017) [6] & 346 & 4.6 \\
David et al. (1999) [9] & 147 & 8.2 \\
Dellaretti et al. (2017) [11] & 105 & 13.3 \\
Feuerberg et al. (1987) [13] & 715 & 3.9 \\
Hollin et al. (1973) [15] & 55 & 5.5 \\
Kivisaari et al. (2004) [16] & 808 & 12.0 \\
Le Roux et al. (1998) [18] & 637 & 5.7 \\
Macdonald et al. (1993) [19] & 78 & 10.3 \\
Meyer et al. (2004) [20] & 384 & 4.9 \\
Proust et al. (1997) [22] & 44 & 2.3 \\
Rauzzino et al. (1998) [23] & 312 & 4.2 \\
Sindou et al. (1998) [24] & 305 & 5.9 \\
\hline
\end{tabular}

*Of which $13.5 \%$ were considered a neck remnant 
Limitations of our evaluation of the intraoperative assessment include its retrospective nature. We cannot exclude the possibility that we have overestimated the number of unexpected residuals. It is possible that the neurosurgeon anticipated a residual but did not document this clearly in the operative report. We attempted to minimize this risk by grouping inconclusive reports with the ones that expected a residual. Furthermore, our study lacks long-term follow-up to demonstrate the natural history of small residuals and their clinical consequences.

\section{Significance of follow-up DSA imaging}

The main goal of postoperative angiography is to eliminate the risk of a rebleed from a residual aneurysm by allowing followup and/or retreatment. Therefore, to decide whether postoperative DSA should be performed routinely, one should balance the risk of a hemorrhage from a (growing) residual with the inherent risk of follow-up DSA and subsequent treatment. The data for this analysis cannot be derived solely from our current study. For an aneurysm residual, the annual risk of regrowth is $2.1 \%$ per year [6] and the annual risk of hemorrhage lies around $0.8 \%$ [13] and 1.5\% [9]. Assuming that the risk of hemorrhage is $1.0 \%$ and is constant throughout the remainder of one's life, the cumulative risk of rupture for a 55 year-old, the average age in our series, with a life expectancy of 83 years is $25 \%\left(1-0.99^{28}\right)$. If we take into account that there is only a $9.6 \%$ chance of an unexpected residual, the rate found in our series, the a priori cumulative lifetime risk of rupture should be estimated at $2.4 \%$ over a period of 28 years. On the other hand, the risk of permanent neurological sequelae from DSA for this patient population is estimated at only $0.13 \%$ [29]. Based on these numbers, we would advocate follow-up DSA imaging unless age, clinical condition, and/or comorbidity sway the balance into another direction or if the residual rate at a specific institution is known to be significantly lower. The latter would require knowledge of ones own residual rate, based on routine DSA analysis. A more detailed and in-depth analysis would require more data on natural history and clinical consequences of residuals and should be part of future research efforts.

\section{Conclusions}

There is lack of consensus regarding the postoperative imaging strategy after intracranial aneurysm clipping in centers throughout the Netherlands. Intra-operative assessment was shown to be insufficient to predict postoperative aneurysm residuals while post-clipping DSA had a substantial yield, at our center. Thus, we believe postoperative imaging should be routine after aneurysm clipping and we suggest that this includes at least one DSA, unless this is not warranted on the basis of patient age, clinical condition and/or comorbidity, or if a center has a documented significantly lower residual rate.

\section{Compliance with ethical standards}

Conflict of interest The authors declare that they have no conflict of interest.

Ethical approval For this type of study, formal consent is not required.

Open Access This article is distributed under the terms of the Creative Commons Attribution 4.0 International License (http:// creativecommons.org/licenses/by/4.0/), which permits unrestricted use, distribution, and reproduction in any medium, provided you give appropriate credit to the original author(s) and the source, provide a link to the Creative Commons license, and indicate if changes were made.

Publisher's note Springer Nature remains neutral with regard to jurisdictional claims in published maps and institutional affiliations.

\section{References}

1. Acevedo JC, Turjman F, Sindou M (1997) Postoperative angiography in surgery for intracranial aneurysm. Prospective study in a consecutive series of 267 operated aneurysms. Neurochirurgie 43: 275-284

2. Akyüz M, Cetin A, Boztuğ N, Kazan S, Tuncer R (2002) Effects of the temporary clipping in aneurysm surgery on the remnant. Acta Neurochir 144:129-136

3. Akyüz M, Tuncer R, Yilmaz S, Sindel T (2004) Angiographic follow-up after surgical treatment of intracranial aneurysms. Acta Neurochir 146:245-250

4. Bernat AL, Clarençon F, André A, Nouet A, Clémenceau S, Sourour NA et al (2017) Risk factors for angiographic recurrence after treatment of unruptured intracranial aneurysms: outcomes from a series of 178 unruptured aneurysms treated by regular coiling or surgery. J Neuroradiol 44:298-307

5. Brown MA, Parish J, Guandique CF, Payner TD, Horner T, Leipzig $\mathrm{T}$ et al (2017) A long-term study of durability and risk factors for aneurysm recurrence after microsurgical clip ligation. J Neurosurg 126:819-824

6. Burkhardt JK, Chua MHJ, Weiss M, Do ASS, Winkler EA, Lawton MT (2017) Risk of aneurysm residual regrowth, recurrence, and de novo aneurysm formation after microsurgical clip occlusion based on follow-up with catheter angiography. World Neurosurg 106:74-84

7. Chen W, Yang Y, Qiu J, Peng Y, Xing W (2008) Sixteen-row multislice computerized tomography angiography in the postoperative evaluation of patients with intracranial aneurysms. Br J Neurosurg 22:63-70

8. Chen W, Yang Y, Qiu J, Peng Y, Xing W (2009) Clinical application of 16-row multislice computed tomographic angiography in the preoperative and postoperative evaluation of intracranial aneurysms for surgical clipping. Surg Neurol 71:559-565

9. David CA, Visteh AG, Spetzler RF, Lemole M, Lawton MT, Partovi S (1999) Late angiographic follow-up review of surgically treated aneurysms. J Neurosurg 91:396-401

10. Dehdashti AR, Binaghi S, Uske A, Regli L (2006) Comparison of multislice computerized tomography angiography and digital subtraction angiography in the postoperative evaluation of patients with clipped aneurysms. J Neurosurg 104:395-403 
11. Dellaretti M, da Silva Martins WC, Dourado JC, Junior FW, Qaudros RS, de Souza Moraes VV et al (2017) Angiographic and epidemiological characteristics associated with aneurysm remnants after microsurgical clipping. Surg Neurol Int 8:198

12. Dolati P, Eichberg D, Wong JH, Goyal M (2015) The utility of dualenergy computed tomographic angiography for the evaluation of brain aneurysms after surgical clipping: a prospective study. World Neurosurg 84:1362-1371

13. Feuerberg I, Lindquist C, Lindqvist M, Steiner L (1987) Natural history of postoperative aneurysm rests. J Neurosurg 66:30-34

14. Hammer A, Steiner A, Kerry G, Ranaie G, Baer I, Hammer CM et al (2017) Treatment of ruptured intracranial aneurysms yesterday and now. PLoS One 12:1-14

15. Hollin SA, Decker RE (1973) Effectiveness of microsurgery for intracranial aneurysms. Postoperative angiographic study of 50 cases. J Neurosurg 39:690-693

16. Kivisaari RP, Porras M, Ohman J, Siironen J, Ishii K, Hernesniemi J (2004) Routine cerebral angiography after surgery for saccular aneurysms: is it worth it? Neurosurgery 55:1015-1024

17. Lee JH, Kim SJ, Cha J, Kim HJ, Lee DH, Choi CG et al (2005) Postoperative multidetector computed tomography angiography after aneurysm clipping: comparison with digital subtraction angiography. J Comput Assist Tomogr 29:20-25

18. Le Roux PD, Elliot JP, Eskridge JM, Cohen W, Winn HR (1998) Risks and benefits of diagnostic angiography after aneurysm surgery: a retrospective analysis of 597 studies. Neurosurgery 42: 1248-1254

19. Macdonald RL, Wallace MC, Kestle JR (1993) Role of angiography following aneurysm surgery. J Neurosurg 79:826-832

20. Meyer B, Urbach H, Schaller C, Baslam M, Nordblom J, Schramm $\mathrm{J}$ (2004) Immediate postoperative angiography after aneurysm clipping - implications for quality control and guidance of further management. Zentralbl Neurochir 65:49-56
21. Nieuwkamp DJ, Vaartjes I, Algra A, Bots ML, Rinkel GJ (2013) Age- and gender-specific time trend in risk of death of patients admitted with aneurysmal subarachnoid hemorrhage in the Netherlands. Int J Stroke 8:90-94

22. Proust F, Toussaint $\mathrm{P}$, Hannequin D, Rabenenoïna C, Le Gars D, Fréger $P$ (1997) Outcome in 43 patients with distal anterior cerebral artery aneurysms. Stroke 28:2405-2409

23. Rauzzino MJ, Quinn CM, Fisher WS 3rd (1998) Angiography after aneurysm surgery: indications for "selective" angiography. Surg Neurol 49:32-40

24. Sindou M, Acevedo JC, Turjman F (1998) Aneurysmal remnants after microsurgical clipping: classification and results from a prospective angiographic study (in a consecutive series of 305 operated intracranial aneurysms). Acta Neurochir 140:1153-1159

25. Sun H, Ma J, Liu Y, Lan Z, You C (2013) Diagnosing residual or recurrent cerebral aneurysms after clipping by computed tomographic angiography: meta-analysis. Neurol India 61:51-55

26. Tines L, Dehdasthi AR, Howard P, Da Costa L, Wallace MC, Willinsky RA et al (2010) Postoperative assessment of clipped aneurysms with 64-slice computerized tomography angiography. Neurosurgery 67:844-854

27. Uysal E, Ozel A, Erturk SM, Kirdar O, Basak M (2009) Comparison of multislice computed tomography angiography and digital subtraction angiography in the detection of residual or recurrent aneurysm after surgical clipping with titanium clips. Acta Neurochir 151:131-135

28. Van Gijn J, Kerr RS, Rinkel GJ (2007) Subarachnoid haemorrhage. Lancet 369:306-318

29. Willinsky RA, Taylor SM, TerBrugge K, Farb RI, Tomlinson G, Montanera W (2003) Neurologic complications of cerebral angiography: prospective analysis of 2,899 procedures and review of the literature. Radiology 227:522-528 